\title{
Broadband Piezocrystal Transducer Array for Non-Destructive Evaluation Imaging Applications
}

\author{
Zhen Qiu, Haoyu Fang, Richard O'Leary, Anthony Gachagan, Alexandru Moldovan \\ Centre for Ultrasonic Engineering, \\ University of Strathclyde, \\ Glasgow, UK \\ zhen.qiu@strath.ac.uk
}

\begin{abstract}
A 32-element, $5 \mathrm{MHz}$ linear array, incorporating PMN-PT 1-3 piezo-polymer composite, has been designed using finite element (FE) modelling. The Elasto-Electric material properties of the PMN-PT samples were characterized and evaluated within the FE model to ensure accurate corroboration. The array configuration and performance were then investigated, including array microstructure, and steering and focusing ability. The matching and backing material selection, array sensitivity and bandwidth were assessed using pulse echo responses. Imaging performance was then undertaken, employing full matrix capture and total focusing method. For performance comparison, a reference array comprising of conventional PZT5H ceramic was also investigated, following the same design criteria. The piezocrystal device provides a bandwidth of $87 \%$ and a sensitivity-bandwidth product improvement of $160 \%$ when compared to the reference PZT5H based array.
\end{abstract}

Keywords-piezocrystal; PMN-PT; broadband; ultrasound linear array; $N D T / E$;

\section{INTRODUCTION}

Bandwidth plays an important role in ultrasound imaging applications such as medical imaging, underwater sonar and inspection in Non-Destructive Evaluation (NDE). Broader operational bandwidth has direct impact on imaging resolution, and can also provide more possibilities in image analysis and post processing, such as the application of split spectrum processing approaches [1]. Industrial manufacturing aiming to achieve better product quality control is having increasing demands in detecting smaller sized defects at an earlier stage, particularly where the propagation medium presents a challenge to signal-to-noise ratio (SNR). Thus broadband transducers with high sensitivity would be highly desirable. However in conventional piezoelectric ceramic transducers, bandwidth and sensitivity are mutually exclusive. The recent development of piezocrystals within (1-x) $\mathrm{Pb}\left(\mathrm{Mg}_{1 / 3} \mathrm{Nb}_{2 / 3}\right) \mathrm{O}_{3^{-}}$ $\mathrm{xPbTiO}_{3}(\mathrm{PMN}-\mathrm{PT})$ family provide a means of advancing both the bandwidth and imaging sensitivity simultaneously [2].

The state-of art piezoelectric material used in ultrasound imaging transducers are generally soft PZT ceramics, e.g., PZT5H, which has an electromechanical coupling coefficient, $k_{33}$ up to 0.75 . Whereas PMN-PT piezocrystals offer ultrahigh electromechanical coupling $k_{33} \approx 0.90$, which can be utilized in 1-3 piezo-polymer composite structures. The resulting broader bandwidth can benefit the imaging resolution not only through application of shorter pulse lengths and spectral post-processing approaches, but also by extending the transducer's effectiveness in applications such as harmonic imaging without challenging manufacturing techniques for higher frequency devices. PMN-PT piezocrystals also offer a higher piezoelectric charge coefficient $d_{33}>2000 \mathrm{pc} / \mathrm{N}$, which is 3-4 times higher than their PZT ceramic counterparts. The resulting high sensitivity provides for a number of benefits in NDE ultrasound imaging: improved SNR; increased penetration depth; and the potential to use lower excitation amplitudes in autonomous robotic inspections[3], [4], [5].

In this work, a linear array transducer is designed and evaluated using commercially available $<001>$ PMN-PT binary piezocrystals. The problem space of developing the array transducer was explored using finite element modelling (FEM) package, PZFlex (OnScale Inc, Cupertino, CA).

\section{MATERIAL CHARACTERIZATION}

The limiting factor of computer aided transducer design is the availability of accurate elastic and piezoelectric constants used to define the materials within the model. Moreover, in the case of piezocrystal devices, non-uniformity between samples needs to be addressed: not only from different manufacturers, but even in different production batches from the same manufacturer [6]. One of the main reasons cited in the literature for such non-uniformity is the loss of $\mathrm{Pb}$ during crystal growth. It has been found that a 1\% PT composition variation may cause up to $50 \%$ variation in some material properties [7].

It is therefore prudent to commence the array design work with material characterization of PMN-PT piezocrystals. Although it would be ideal to obtain a complete set of ElastoElectric properties, full matrix characterization would require multiple samples of multiple geometries as stated in IEEE standard, and could be both time and cost consuming. Thus we have adopted a combined approach incorporating both experimental IEEE resonance methods and virtual 
prototyping. Having access to 10 sample plates with a geometry of $15 \times 15 \times 1 \mathrm{~mm}$, we measured the impedance spectrum of the samples and extracted the relevant parameters: stiffness constants $c^{E}{ }_{33}$, piezoelectric coefficients $e_{33}$, dielectric permittivity $\varepsilon_{r 33}^{S}$ and mechanical factor $Q$, from the thickness-extensional (TE) resonance. The measurements were averaged and then imported into a proprietary calculation scheme to determine the full set of material properties required by PZFlex - other missing parameters being acquired from literature values [6], [9]. Being aware of the non-consistency of the parameters obtained, we imported the parameters into PZFlex model. The comparison of the simulated and measured impedance spectra was then used to ensure appropriate data set had been determined. The resulting properties of PMN-PT piezocrystals utilized in the PZFlex simulation are listed in Table 1.

Table 1 Relevant bulk Material properties for PMN-PT piezocrystal used in FEM simulation

\begin{tabular}{cccccc}
\multicolumn{6}{c}{ Elastic stiffness constants: $C^{E}{ }_{i j}\left(10^{10} \mathrm{~N} / \mathrm{m}^{2}\right)$} \\
\hline$c^{E}{ }_{11}$ & $c^{E}{ }_{12}$ & $c^{E}{ }_{13}$ & $c^{E}{ }_{33}$ & $c^{E}{ }_{44}$ & $c^{E}{ }_{66}$ \\
11.3 & 9.76 & 10.0 & 11.5 & 6.6 & 6.0 \\
\hline \multicolumn{5}{l}{ Piezoelectric coefficients: $e_{i j}(C / m 2)$ and dielectric permittivity $\varepsilon_{r i j}^{S}$} \\
\hline$e_{31}$ & $e_{15}$ & $e_{33}$ & $\varepsilon_{r 33}^{S}$ & $\varepsilon_{r l 1}^{S}$ \\
-5.9 & 10 & 21.5 & 925 & 1000 \\
\hline \multicolumn{5}{c}{ Mechanical factor and Density $\left(\mathrm{kg} / \mathrm{m}^{3}\right)$} \\
\hline \multicolumn{5}{c}{$\rho$} \\
\hline
\end{tabular}

Fig. 1 presents the simulated and measured impedance magnitude spectra of a bulk plate of piezocrystal. Good agreements are achieved at both high and low frequency resonances. It should be noted that the $2^{\text {nd }}$ lateral resonance shown in Fig. 1 (b) is likely related to the vibration around the axis. Although the constant $c_{66}^{E}$ could be adjusted to higher value to maximize the corroboration, we decided not to do so, as only the fundamental resonances are considered when using IEEE resonance methods.

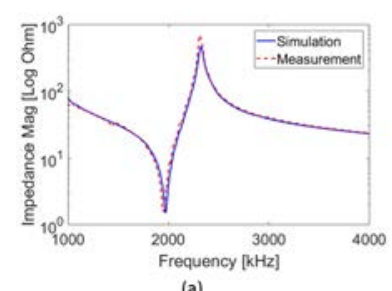

(a)

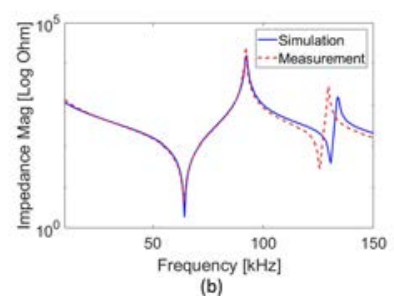

Fig. 1 Material properties valuation using impedance spectrum of a $15 \mathrm{x}$ $15 \times 0.98 \mathrm{~mm}$ square plate at (a) thickness extensional resonance; (b) fundamental lateral resonance.

From the set of 10 samples under consideration, the average electrical resonance frequency, $f_{e}$, and mechanical resonance frequency, $f_{m}$, at fundamental TE resonance are $1952 \mathrm{kHz}$ and $2313 \mathrm{kHz}$, respectively, with same standard deviation of $0.87 \%$. This is in line with the thickness variations we found across the range of samples

\section{TRANSDUCER DESIGN AND DEVELOPMENT}

\section{A. 1-3 composite geometry}

PMN-PT piezocrystals need to be utilized in 1-3 piezopolymer composite structures to take advantage of its ultrahigh through-thickness coupling coefficient, $k_{33}$. The micro-structure of the 1-3 composite was assessed firstly, taking practical limits in manufacturing into account. Since the composite will be manufactured using dice-and-fill technique, we proceeded dicing trials using a $25 \mathrm{um}$ hubbed nickel/diamond blade (DISCO HI-TEC EUROPE GmbH, Germany) and a dicing saw (MicroACE Series 3, Loadpoint, $\mathrm{UK})$. The actual dicing kerf in the piezocrystal is $28 u \mathrm{~m}$ when cut to a depth of $400 u \mathrm{~m}$. The dicing pitch is then determined to be $96 u \mathrm{~m}$ for $50 \%$ volume fraction. Hardset polymer (Epofix, Struers A/S, Denmark) was chosen as the passive filler to the composite. The composite has a thickness of $226 u \mathrm{~m}$ to operate at $5 \mathrm{MHz}$, with a pillar aspect ratio of 0.3 . The FE derived electrical impedance spectrum is shown in Fig 2, with $f_{e}=5.0 \mathrm{MHz}, f_{m}=8.0 \mathrm{MHz}$ and the resulting thickness mode coupling coefficient, $k_{t}$, calculated to be 0.81 . The longitudinal velocity for the modeled geometry was $3616 \mathrm{~m} / \mathrm{s}$ (calculated from $f_{m}$ ) and the overall density of composite was $4610 \mathrm{~kg} / \mathrm{m}^{3}$, leading to 16.7 MRayls as the acoustic impedance of the composite.

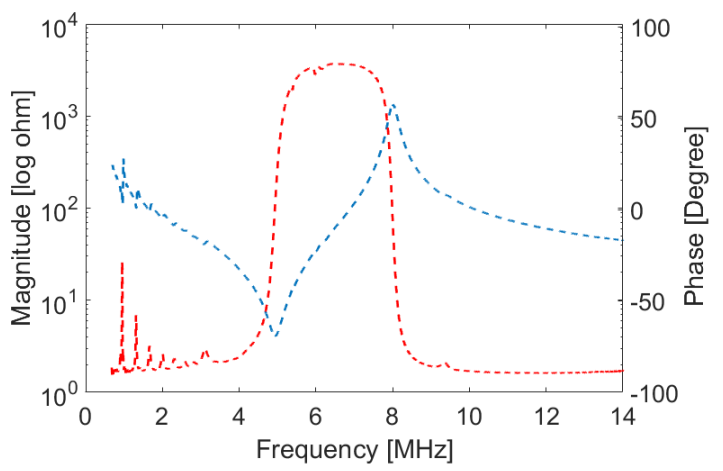

Fig. 2 FE derived electrical impedance spectrum of 1-3 piezocrystal composite operating in air

\section{B. Array element geometry}

We have set the array element pitch as $400 u \mathrm{~m}$, with 270 $u \mathrm{~m}$ element width and $130 u \mathrm{~m}$ element gap, to ensure that each array element comprises 3 pillars in array azimuth direction. For a center frequency of $5 \mathrm{MHz}$, this pitch is equivalent to approximately $0.75 \lambda$ in Plexiglass and $0.35 \lambda$ in steel. For the case where the array is coupled with angled wedge made of Plexiglass, the array is considered as undersampled according to the classical half-wavelength rule [10]. In order to analyze the element pitch effect on array performance, a model of a 32 elements linear array was built in Matlab (R2017a, MathWorks) using Huygens-Fresnel principle. The focus was set to be $50 \mathrm{~mm}$ in Plexiglass, within the transducer near field, and the beam was steering from -45 to 45 degrees. The results presented in Fig. 3 indicate that when the ultrasound beam was steered 20 degree off the array central axis, the side lobe intensity reaches to its maximum and a strong grating lobe appears about 85 degree away from the main beam and with its amplitude exceeding $-10 \mathrm{~dB}$ of the main beam.

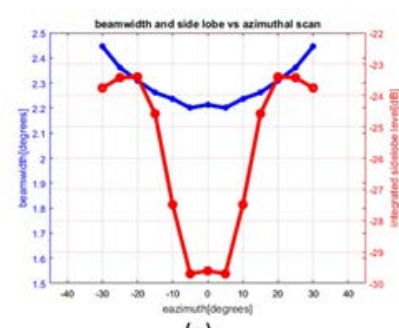

(a)

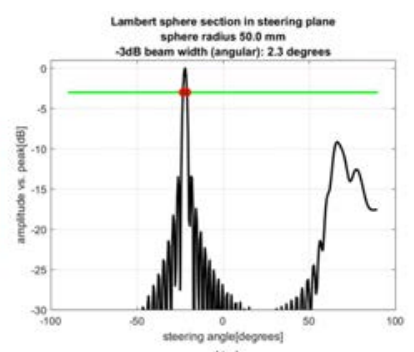

(b)
Fig. 3 Simulated beam profile when focused at $50 \mathrm{~mm}$ (a) beam width and averaged side lobe level during steering (b) main beam and grating lobe 


$$
Z_{m}=Z_{C}{ }^{1 / 3} \cdot Z_{L}{ }^{2 / 3}
$$

This is further evident in the FEM results shown in Fig. 4. The same array configuration was modelled in PZFlex, coupled to a steel block via a $30^{\circ}$ Plexiglass wedge. Time delays were applied to individual elements to steer the beam 20 degrees off the array axis, and focused $30 \mathrm{~mm}$ deep into the steel block. The grating lobe is visible as expected, however, its effects on imaging quality could be suppressed in practice by introducing specially designed wedge with an integrated absorbing material. Nevertheless, we set the steering angle limit of the array to be 20 degrees at this stage. The angular coverage in the steel block for the inspection scenario is illustrated in Fig. 4 would be 90 to 20 degrees according to Snell's law, when beam is steered from 0 to 20 degrees with respect to the array normal. If using normalincidence direct-contact configuration to perform total focusing method (TFM) imaging, $0.75 \lambda$ array requires fewer elements to populate an aperture of a certain size, and the resulting full matrix capture (FMC) data set is $57 \%$ less than an equivalent half lambda array. Recent research has advised an upper aperture angle limit should be imposed in the algorithm to ensure grating lobe artifacts are eliminated [11]. The post-processing algorithms are not investigated here.

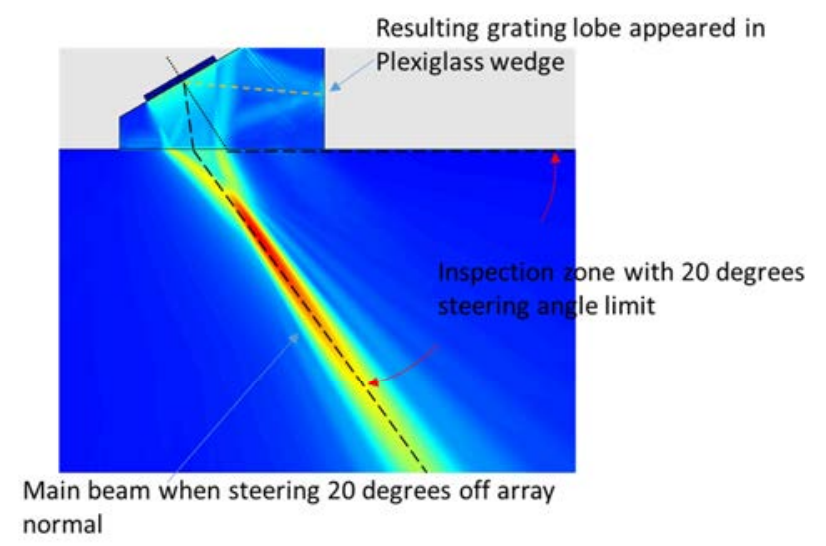

Fig. 4 FE derived acoustic pressure field in steel block with the ultrasound beam steered 20 degree off the array axis via Plexiglass wedge.

\section{Matching layer and Backing layer}

The selection of matching layer and backing layer was assessed with a model consisting of one array element, operating in symmetrical boundary condition in azimuthal direction and free boundary condition in elevation direction. A single matching layer was introduced to acoustically couple the transducer to the Plexiglass wedge, which has an acoustic impedance $\left(\mathrm{Z}_{\mathrm{L}}\right)$ of 3.16 MRayls. In order to optimize the matching material selection, Equation 2 (a) and (b) were both used to predict the matching section, using transmission line theory and KLM equivalent circuit model, respectively. The ideal impedance of matching layer from these calculations are 7.26 MRayls and 5.5 MRayls. Therefore the hardset polymer (RX771C(NC)/CY1300, Robnor Resin Ltd, UK) filled with $3 \mu \mathrm{m}$ alumina powder using $70 \%(Z=6.9$ MRayls $)$ and $50 \%(Z=4.7$ MRayls $)$ weight fraction were chosen for investigation. Two approaches are used to determine the thickness of the matching layer as either the $1 / 4 \lambda$ thick at central frequency or $0.35 \lambda$ at anti-resonance frequency [12]. That results in four options of matching layer as listed in Table 2.

$$
Z_{m}=\sqrt{Z_{C} \cdot Z_{L}}
$$

Three backing materials were also studied and are listed in Table 2. The backing thickness was set to be $5 \mathrm{~mm}$ in the model but with an absorbing boundary condition in order to reduce computational time. Thus, the thickness information is not included in the Table.

Table 2 Matching and backing layers characteristics

\begin{tabular}{cccc} 
& Material & $\mathrm{Z}_{\mathrm{AC}}$ (MRayls) & Thickness (um) \\
\hline$M 1$ & $70 \% \mathrm{Al}_{2} \mathrm{O}_{3}+\mathrm{HS}$ & 6.9 & 126 \\
$M 2$ & $50 \% \mathrm{Al}_{2} \mathrm{O}_{3}+\mathrm{HS}$ & 4.7 & 113 \\
$M 3$ & $70 \% \mathrm{Al}_{2} \mathrm{O}_{3}+\mathrm{HS}$ & 6.9 & 146 \\
$M 4$ & $50 \% \mathrm{Al}_{2} \mathrm{O}_{3}+\mathrm{HS}$ & 4.7 & 132 \\
\hline Back15 & $15 \%$ tungsten + HS & 7.1 & \\
Back20 & $20 \%$ tungsten + HS & 8.6 & \\
Back25 & $25 \%$ tungsten + HS & 10 & \\
\hline
\end{tabular}

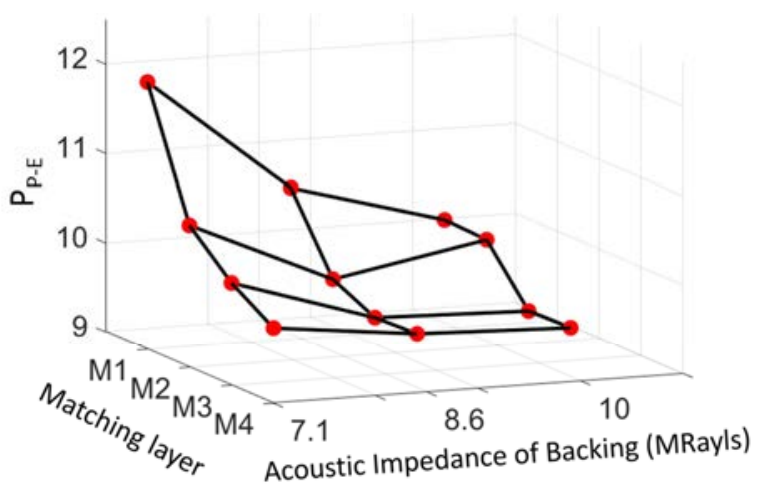

Fig. 5 Sensitivity-bandwidth product from array element acoustically coupled to matching and backing materials

The pulse-echo response was simulated to evaluate the element performance with the combination of matching and backing materials detailed in Table 2. To consider time domain sensitivity and frequency domain bandwidth of the pulse echo response of the array, we used a figure of merit to describe the 'sensitivity-bandwidth product': $P_{P-E}=V \cdot B$, where $V$ is the time domain amplitude of the reflected echo signal from Plexiglass-steel interface recorded for relative element sensitivity comparisons, $B$ is the $-6 \mathrm{~dB}$ bandwidth in $\mathrm{MHz}$ of the pulse-echo spectrum. The results are presented in Fig. 5, and the optimized combination of matching and backing layers are matching layer 'M1', calculated using the guideline of $0.35 \lambda$ thick at anti-resonance frequency, and backing layer 'Back15'. A broad bandwidth of $87 \%$ at $-6 \mathrm{~dB}$ was predicted for the piezocrystal array. For comparison purpose, a similar model was built with PZT5H piezoelectric ceramic. Same design approaches and criteria were used when optimizing the ceramic array characteristics, resulting a backing layer 'Back25' and matching layer 'M3'. The sensitive-bandwidth product of piezocrystal array is $160 \%$ of the reference ceramic array.

\section{TFM Imaging}

The array's imaging ability was assessed in the FEM suite in a normal-incidence direct-contact configuration with a steel block containing 2 side drilled hole defects of diameter of $\lambda / 2$ of the longitudinal wave and a $2 \lambda$ separation distance. In this imaging evaluation model, the input forcing function for each array element was the pulse echo response from a separate model containing one array element, which 
was directly coupled to the steel block. Since the matching layers were chosen to match the composite to Plexiglass, it is not ideal to analyze the pulse-echo responses when the arrays were coupled to steel directly, especially for sensitivity. However, as both arrays were assessed under the same operation condition, a valuable comparison can still be achieved.

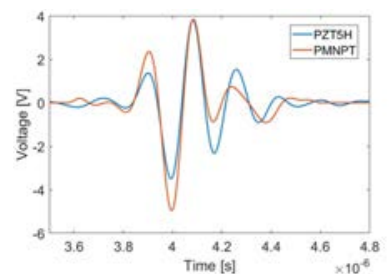

(a)

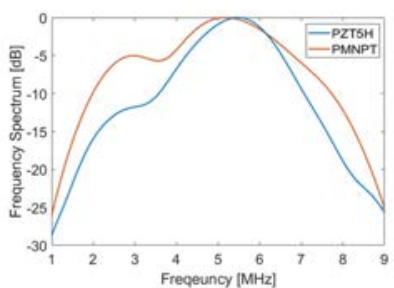

(b)
Fig. 6 FE derived pulse-echo responses from Piezcrystal array element and referenced ceramic array element which are used as input pulses for generating TFM images

The pulse-echo responses from the one element model are presented in Fig. 6, and as noted earlier, used as input load signals to the array elements for imaging model. The stress signals at the dummy element surfaces were recorded to form a FMC dataset and subsequently generate the TFM images presented in Fig.8. The $-6 \mathrm{~dB}$ measurements on contour plots of the same results are $1.32 \mathrm{~mm}$ vs $1.34 \mathrm{~mm}$ in lateral direction for piezocrystal array and ceramic array, respectively, and $0.7 \mathrm{~mm}$ vs. $0.9 \mathrm{~mm}$ in axial direction.

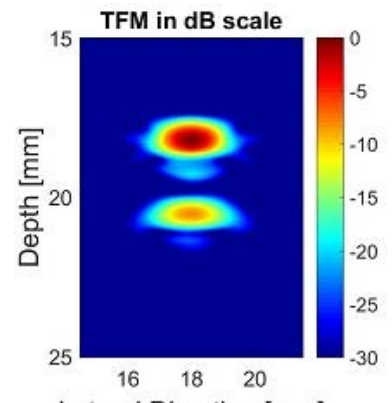

(a)

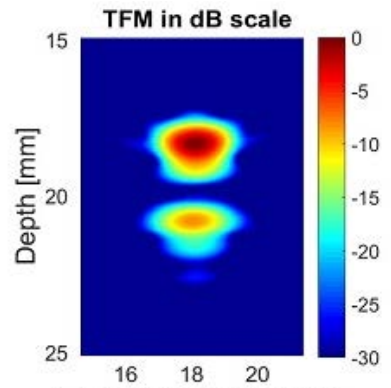

Lateral Direction [mm]

(b)
Fig. 7 Predicted TFM images of two SDHs $(0.6 \mathrm{~mm}, \sim 1 / 2 \lambda$ dia.) from: (a) piezocrystal array and (b) ceramic array.

\section{CONCLUSION}

In this work, a $5 \mathrm{MHz}, 32$ elements linear array utilizing PMN-PT binary piezocrystal was designed using finite element modeling. The bulk material properties were characterized and then, the sensitivity-bandwidth product was used as design criteria to select matching and backing layers. The focusing and steering ability of the proposed array were studied and the maximum steering angle was set to be 20 degree due to an under sampled array pitch when operating into Plexiglass. The predicted bandwidth of the piezocrystal array is $87 \%$ at $-6 \mathrm{~dB}$ and also demonstrated a sensitivity-bandwidth product improvement of $160 \%$ when compared to a reference ceramic array. Finally, the FE derived TFM image also demonstrates that the piezocrystal array has enhanced image resolution and hence, better defect sizing ability.

\section{ACKNOWLEDGMENTS}

This work has been carried out under the auspices of the UK Research Centre for NDE (RCNDE) (EPSRC Grant $\mathrm{EP} / \mathrm{L} 022125 / 1)$.

\section{REFERENCES}

[1] P. Karpur and O. J. Canelones, "Split spectrum processing: a new filtering approach for improved signal-to-noise ratio enhancement of ultrasonic signals," Ultrasonics, vol. 30, no. 6, pp. 351-357, 1992.

[2] S. Zhang and T. Shrout, "Relaxor-PT single crystals: observations and developments.," IEEE TUFFC, vol. 57, no. 10, pp. 2138-46, Oct. 2010.

[3] R. Zhang, B. Jiang, and W. Cao, "Elastic, piezoelectric, and dielectric properties of multidomain $0.67 \mathrm{~Pb}(\mathrm{Mg}$ [sub $1 / 3] \mathrm{Nb}$ [sub 2/3])O[sub 3]-0.33PbTiO[sub 3] single crystals," J. Appl. Phys., vol. 90, no. 7, p. 3471, 2001.

[4] S. Zhang and F. Li, "High performance ferroelectric relaxor-PbTiO3 single crystals: Status and perspective," J. Appl. Phys., vol. 111, no. 3 , p. 31301, 2012.

[5] X. Jiang et al., "Single crystal piezoelectric composite transducers for ultrasound NDE applications," Proc. SPIE, vol. 6934, p. 69340D69340D-10, Mar. 2008

[6] Z. Qiu, et al, "Characterization of piezocrystals for practical configurations with temperature- and pressure-dependent electrical impedance spectroscopy.," IEEE TUFFC, vol. 58, no. 9, pp. 1793803, Sep. 2011.

[7] W. Jiang, et al, "Characterization of piezoelectric materials with large piezoelectric and electromechanical coupling coefficients," Ultrasonics, vol. 41, no. 2, pp. 55-63, Mar. 2003.

[8] "An American National Standard IEEE Standard on Piezoelectricity," ANSI/IEEE STd 176-1987, 1988.

[9] J. Luo, "Elastic, piezoelectric and dielectric properties of PIN-PMN-PT crystals grown by Bridgman method," in 2008 IEEE IUS, 2008, pp. 261-264.

[10] S.-C. Wooh and Y. Shi, "Optimum beam steering of linear phased arrays," Wave Motion, vol. 29, pp. 245-265, 1999.

[11]P. D. Wilcox and J. Zhang, "Quantification of the Effect of Array Element Pitch on Imaging Performance," IEEE TUFFC, vol. 65, no. 4, pp. 600-616, 2018.

[12] R. E. McKeighen, "Design guidelines for medical ultrasonic arrays," in Proc. SPIE 3341, Medical Imaging: Ultrasonic Transducer Engineering, 1998, pp. 2-18.

[13] C. Holmes, "Post-processing of the full matrix of ultrasonic transmitreceive array data for non-destructive evaluation," NDT E Int., vol. 38 , no. 8, pp. 701-711, Dec. 2005. 